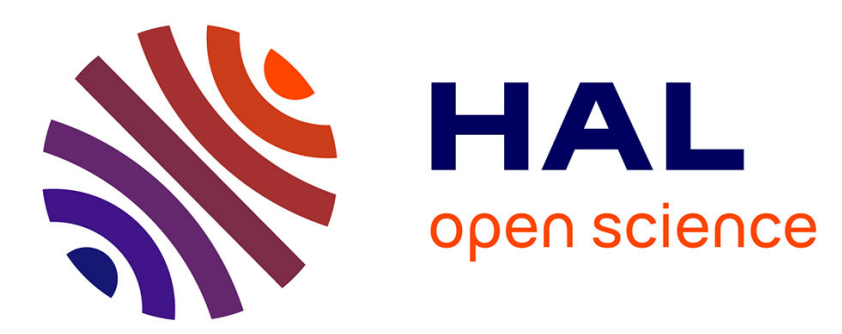

\title{
Influence of electric polarization on Coulomb blockade in a super-paraelectric clusters assembly
}

Brice Kengni-Zanguim, Nabil Najjari, Bernard Doudin, Yves Henry, David Halley

\section{- To cite this version:}

Brice Kengni-Zanguim, Nabil Najjari, Bernard Doudin, Yves Henry, David Halley. Influence of electric polarization on Coulomb blockade in a super-paraelectric clusters assembly. Applied Physics Letters, 2019, 115 (26), pp.262901. 10.1063/1.5128846 . hal-02996509

\section{HAL Id: hal-02996509 \\ https://hal.science/hal-02996509}

Submitted on 10 Nov 2020

HAL is a multi-disciplinary open access archive for the deposit and dissemination of scientific research documents, whether they are published or not. The documents may come from teaching and research institutions in France or abroad, or from public or private research centers.
L'archive ouverte pluridisciplinaire HAL, est destinée au dépôt et à la diffusion de documents scientifiques de niveau recherche, publiés ou non, émanant des établissements d'enseignement et de recherche français ou étrangers, des laboratoires publics ou privés. 


\section{Influence of electric polarisation on Coulomb blockade in a super- paraelectric clusters assembly}

Brice Kengni-Zanguim, Nabil Najjari, Bernard Doudin, Yves Henry and David Halley IPCMS, 23 rue du Loess, BP 43, F-67034 Strasbourg Cedex 2, France

\section{$\underline{\text { Abstract }}$}

We study electron transport in an assembly of epitaxial $\mathrm{Cr}_{2} \mathrm{O}_{3}$ nanoparticles embedded in a $\mathrm{MgO}$ tunnel barrier: a non-usual variation in the Coulomb blockade charging energy is observed as a function of the size of the clusters. In striking contrast to the expected increase of charging energy when decreasing the clusters size, an almost constant behaviour is observed. We argue here that the spontaneous super-paraelectric moment carried by the clusters core is the origin of this unusual behaviour since it drives the dielectric constant in this clusters assembly. This phenomenon could be exploited to fabricate devices with single valued Coulomb blockade energy despite a statistical dispersion in the clusters size.

\section{$\underline{\text { Text }}$}

Scaling electronic devices down to nanometric dimensions often leads to electrostatic repulsive effects inside a single nanoparticle or epitaxial cluster, strongly modulating the electron conductance via the clusters as a function of the applied bias voltage. This Coulomb blockade (CB) effect was observed in many devices including conductive clusters -metallic, organic or semi-conductive - embedded in an insulating matrix ${ }^{[1,2]}$ and is extensively exploited for developing single electron transistors (SET) devices. ${ }^{[1,3,4]} \mathrm{CB}$ occurs as the energy $\Delta E=\mathrm{e}^{2} / C$ needed to add an electron on a particle of capacitance $C$ becomes significantly larger than the thermal and quantum fluctuation energies. With $C \approx 4 \pi \varepsilon_{0} \varepsilon_{\mathrm{r}} l$ for 
particles of length scale $l$, this energy scales inversely with the size of the particle and becomes dominant when $l$ is reduced down to a few $\mathrm{nm}$.

The dielectric environment (of constant $\varepsilon_{\mathrm{r}}$ ) of the particles can however also possibly change with the length scale of the system, and can be significantly modified though external parameters, as an applied electric field. This can be the case for ferroelectric systems for which there is a growing interest at the nanoscale, since they could be the key to new nanometric devices, for instance high-density non-volatile memories. ${ }^{[5]}$ Preserving a ferroelectric state when decreasing the material dimensions down to a few nanometers is challenging $^{[6,7]}$ but has been recently reported $^{[8]}$ in several high quality ferro-electric oxides $^{[9,10]}$. The issue of $\mathrm{CB}$ effects combined with a dielectric medium polarisation was theoretically discussed by of Udalov et al. ${ }^{[11,12]}$ with one experimental report we are aware of. ${ }^{[13]}$ Those works considered composite devices with nanometric conducting clusters embedded in a ferro-electric matrix, or above a ferro-electric gate. It turns out that the coupling between $\mathrm{CB}$ effects and the surrounding electric polarisation is far from being negligible and induces a large modification of the conductance Coulomb diamonds in the clusters.

We recently reported ${ }^{[14-16]}$ a system, made of granular $\mathrm{Cr}_{2} \mathrm{O}_{3}$ nanoparticles embedded in a $\mathrm{MgO}$ insulating matrix, where, schematically, the in-plane interface of flat chromium oxide nanometric epitaxial islands with insulating $\mathrm{MgO}$ are in a conducting state whereas the core of the clusters is insulating and carries a spontaneous electric moment. ${ }^{[14,17-19]}$ It provides a unique system with a metallic fraction playing the role of a Coulomb island, next to a dielectric of high polarizability. We show here that the Coulomb charging energy $\Delta \mathrm{E}$ is nearly size invariant, in striking contrast from the expectations of the size dependence of the capacitance of an island. As the nanoparticles also influence the dielectric environment through their insulating fraction, we discuss here how it can impact the charging energy, 
explaining quantitatively how it makes it size independent. This phenomenon is highly appealing for developing new devices exploiting $\mathrm{CB}$ effects with a well-defined $\Delta E$ values, providing a clear electrical spectroscopy signature that is robust versus the particles size statistics.

The tunnel junctions are grown by e-beam gun on an $\mathrm{MgO}$ [001] substrate by molecular beam epitaxy in ultra-high vacuum: the bottom $\mathrm{Fe}$ electrode is deposited at $100^{\circ} \mathrm{C}$ and annealed at $500^{\circ} \mathrm{C}$ for one hour yielding an atomically flat surface. A $3 \mathrm{~nm} \mathrm{MgO}$ layer is then deposited at $100^{\circ} \mathrm{C}$, followed by the $\mathrm{Cr}$ layer -nominal thickness $\mathrm{t}_{\mathrm{Cr}}$ - and $3 \mathrm{~nm} \mathrm{MgO}$ at $200^{\circ} \mathrm{C}$. Chromium crystallizes in a centred cubic structure with a $\mathrm{Cr}[100](100) / /$ $\mathrm{MgO}[110](100)$ epitaxial relationship, and follows a Volmer-Weber growth mode ${ }^{[20,21]}$ leading to the appearance of flat islands embedded in the smooth $\mathrm{MgO}$ barrier. The stack (Fig. (a)) is then annealed at $500^{\circ} \mathrm{C}$ to achieve a complete oxidation of $\mathrm{Cr}$ into $\mathrm{Cr}_{2} \mathrm{O}_{3} .^{[14]}$ The top electrode is made of $5 \mathrm{~nm}$ Fe followed by $20 \mathrm{~nm}$ Co capped with Pd. Wedge samples with a varying $\mathrm{t}_{\mathrm{Cr}}$ are obtained by progressively masking the sample during deposition of chromium (Fig.1(b)), providing a straightforward method to vary continuously the average cluster size value on a single processed batch. A complete structural analysis was previously detailed ${ }^{[14]}$ : Chromium was shown to form $\mathrm{Cr}_{2} \mathrm{O}_{3}$ clusters inside the $\mathrm{MgO}$ barrier, with a $2 \mathrm{~nm}$ height and 4-8 nm width. X-ray Absorption Spectroscopy measurements on such clusters indicated a complete oxidation of chromium, mostly into the corundum $\mathrm{Cr}_{2} \mathrm{O}_{3}$ phase (about $90 \%$ ), whose c-axis was in the plane, and (less than $10 \%$ ) into an oxygen-rich phase close to metallic $\mathrm{CrO}_{2}$ probably located at the edge of the clusters. The metallic part of the clusters is possibly due to the oxygen rich $\left(\mathrm{VO}_{2}\right.$-like) oxide but the large strained-induced modification of $\mathrm{Cr}_{2} \mathrm{O}_{3}$ relative to the bulk - see for instance the appearance of a magnetization- could also make that $\mathrm{Cr}_{2} \mathrm{O}_{3}$ locally turns into a conducting phase. Tunnel junction devices in the microns range of lateral 
size were obtained through successive U.V. photolithography, $\mathrm{Ar}^{+}$ions milling, and $\mathrm{SiO}_{\mathrm{x}}$ and Ti/Au deposition steps as in Ref. [14]. Electron transport measurements were performed in a $\mathrm{He}_{4}$ static cryostat, in a four-wire configuration. The conductance $G$ per junction surface unit A (Fig. 1c) increases linearly with the nominal deposited chromium thickness. This is consistent with our previous observations ${ }^{[14]}$ : the density of $\mathrm{Cr}_{2} \mathrm{O}_{3}$ clusters - in the order of $10^{4} / \mu \mathrm{m}^{2}$ - and their thickness $d$ - equal to $2 \mathrm{~nm}$ - do not change with the amount of deposited chromium, as was also pointed by Koda and coworkers ${ }^{[20,21]}$ on similar systems. As a consequence, only the area $S$ of individual clusters varies, proportionally to $t_{C r}$, explaining the linear conductance variation observed in Fig.1(c): the direct tunnelling of electrons between bottom and top electrodes being ruled out, the tunnelling takes probably place via the clusters interfaces whose surface is proportional to $t_{C r}$. A sketch of an individual cluster is given in Fig. 1(d), supposing a metallic phase at the edge of the cluster. Notice that in an alternative hypothesis the metallic part could be located closer to the cluster core.

The I(V) curves measured at $6.2 \mathrm{~K}$ - see Fig. 2(a)- are nearly symmetric in voltage and do not exhibit striking features, contrary to the second derivative curves (Fig. 2(b)) which present resonance peaks almost symmetric in positive and negative voltages. The two well-defined peaks in the positive and negative voltage ranges are located at voltages in the order of \pm 40 $\mathrm{meV}$ and $\pm 130 \mathrm{meV}$. A simulation of the expected peaks in $\frac{\partial^{2} I}{\partial V^{2}}$ curves due to a $\mathrm{CB}$ effect in such clusters is given in the inset of Fig. 2(b): this simulation, based on an orthodox theory ${ }^{[1,14]}$ evaluates the electronic occupation probability on a cluster and, taking into account the tunnelling probability through both $\mathrm{MgO}$ tunnel barriers and yields the conductance at a given voltage. A background charge of $\mathrm{Q}=0.28$ electron for $\mathrm{t}_{\mathrm{Cr}}=0.71 \mathrm{~nm}$ reflects the global environment charge in the vicinity of an individual cluster or an intrinsic electric polarisation. The $\mathrm{Cr}_{2} \mathrm{O}_{3}$ clusters diameters follow a Gaussian distribution law centered at $7.1 \mathrm{~nm}$ (with a 
standard deviation of $1.2 \mathrm{~nm}$ ), extracted from experimental observations. ${ }^{[14]} \mathrm{We}$ do not reproduce the background of the whole curve, but the agreement with the shape of peaks in experimental I"(V) curves derivatives confirms that we deal with a CB effect. ${ }^{[14]}$

The positive voltage at which we observe the experimental first peak in $\frac{\partial^{2} I}{\partial V^{2}}$ curves varies slowly with a slight, linear decrease as a function of $\mathrm{t}_{\mathrm{Cr}}$ (Fig. 3). Relatively to the first peak, the voltage corresponding to the second peak in $\frac{\partial^{2} I}{\partial V^{2}}$ curves follows nearly the same linear variation as a function of $\mathrm{t}_{\mathrm{Cr}}$, which implies that the energy difference $\Delta E$ between the two states ( $n$ or $n+1$ electrons on the cluster) is nearly independent of the clusters size. This is highly intriguing as this value should decrease with increasing the nominal chromium thickness, i.e. when increasing the capacitance associated to the cluster. Indeed, defining $\varepsilon r$ as the relative dielectric constant of the environment, made essentially of insulating $\mathrm{Cr}_{2} \mathrm{O}_{3}$, and $S$ as the clusters individual area, the energy $\Delta E$ should schematically vary as the inverse of $C=$ $\varepsilon_{0} \varepsilon_{\mathrm{r}} S / d$ for our disc-shaped particles. ${ }^{[1,14]}$ This should imply a noticeable decrease of $\Delta E$ from $\mathrm{t}_{\mathrm{Cr}}=0.08 \mathrm{~nm}$ to $\mathrm{t}_{\mathrm{Cr}}=0.78 \mathrm{~nm}$, which is here clearly not experimentally observed. This expectation is confirmed in Fig. 4(a): a simulation using the orthodox model, for clusters of varying diameters, and an average zero environment charge, shows a large decrease of $\Delta E$ with $\mathrm{t}_{\mathrm{C}}$. The counter-intuitive experimental behaviour thus suggests that a more subtle mechanism is at play.

We propose that the presence of the individual electric dipole $\mathbf{P}$ in the clusters ${ }^{[14,15,17]}$

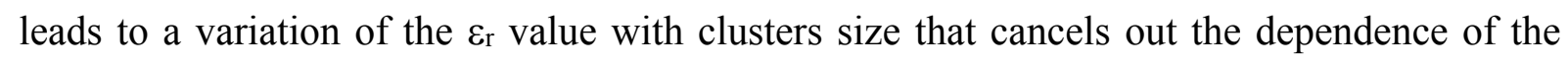
capacitance on the clusters surface area. Since the dielectric constant writes $\varepsilon_{0} \varepsilon_{\mathrm{r}}=\varepsilon_{0}(1+\chi)$, were $\chi$ is the electric susceptibility, we estimate $\chi$, the contribution of the weak spontaneous electric polarisation to $\chi$ in our particular granular systems. As was shown in our former 
works $^{[14,15]}$ each cluster carries a - weak- spontaneous electric dipole $\mathbf{P}-$ in the order of $5.10^{-}$ ${ }^{29}$ C.m- induced by the large epitaxial strain - which probably explains the large, voltagedepend, magnetic anisotropy reported in Ref[16]- . This electric polarization could only be indirectly evaluated ${ }^{[14,15]}$ as direct classical observations - as capacitance measurements- are not sensitive enough for such low polarization values. Our previous findings ${ }^{[14]}$ suggested that the individual electric dipole modulus carried by those clusters followed a complex behaviour as a function of the clusters size, because of two competing effects: increasing the clusters area induces a relaxation of epitaxial strain, and thus a lower electric polarisation, but on the other hand the clusters volume gets larger. Within the $\mathrm{t}_{\mathrm{Cr}}$ range considered in the present work [0.08 $\mathrm{nm}$ to $0.78 \mathrm{~nm}]$, we therefore observe a relatively low change of the magnitude of $\|\boldsymbol{P}\|$, that we neglect for simplicity in the following discussion. Moreover, the low anisotropy energy enables the electric dipoles to rotate due to thermal fluctuations: the whole clusters assembly is in a super-paraelectric state. ${ }^{[22-24]}$ The alignment of the electric dipoles along the applied electric field is thus described by a Langevin law as a function of an applied electric field. $^{[14,15]}$

The estimate of $\chi d$ relates to the average electric dipole along $\mathrm{z}$ of an individual cluster, $P_{\mathrm{z}}$, when applying an electric field $E$ along this direction (perpendicularly to the tunnel barrier), which favors the alignment of the individual permanent electric dipoles along this direction. This polarisation can be written: $\frac{P_{z}}{V}=\varepsilon_{0} \chi_{d} E$ where $V$ is the volume of an individual cluster. It relates to the magnitude of the spontaneous dipole $\|\boldsymbol{P}\|$ by $P_{z}=\|P\| \times L\left(\frac{\|P \cdot\| E}{k_{B} T}\right)$ where $\mathrm{L}$ is the Langevin function defined as $\mathrm{L}(\mathrm{x}) \equiv 1 / \mathrm{x}-1 / \tanh (\mathrm{x}) . \mathrm{We}$ make an approximation, valid for $x<1$, by supposing $L(x) \approx \frac{x}{3}$ (in fact, for an applied voltage 
of $0.05 \mathrm{~V}$, we can reach $\mathrm{x}=3$ : this means that we will here, for simplicity sake, slightly overestimate the slope of $\mathrm{L}(\mathrm{x})$ and the subsequent electric susceptibility).

Within this approximation, $P_{z}=\frac{\|P \cdot\|^{2} E}{3 k_{B} T}$ and thus: $\chi_{d}=\frac{P_{z}}{V \cdot E \cdot \varepsilon_{0}}=\frac{\|P .\|^{2}}{3 k_{B} T \cdot V \cdot \varepsilon_{0}}$

Taking our experimental findings $d=2 \mathrm{~nm}$, and a characteristic $\|P\|$ value of $5.10^{-29}$ C.m ${ }^{[14]}$, we obtain, at $\mathrm{T}=6.2 \mathrm{~K}$, a value of $\chi_{d}$ close to 50 , much larger than unity - and the corresponding $\varepsilon_{\mathrm{r}}$ value of bulk $\mathrm{Cr}_{2} \mathrm{O}_{3}$ equal to $9^{[25]}$-. The contribution of $\chi_{\mathrm{d}}$ therefore dominates the dielectric constant and: $\varepsilon_{r} \approx \frac{\|P .\|^{2}}{3 k_{B} T \cdot V \cdot \varepsilon_{0}}$

Finally, using our estimate for the capacitance of cylindrical clusters, we obtain: $\Delta E=\frac{3 e^{2} k_{B} T \cdot V \cdot d}{S \cdot\|P\|^{2}}=\frac{3 e^{2} k_{B} T \cdot d^{2}}{\|P\|^{2}}$ This expression can be simplified if we define the equivalent electric charge due to the spontaneous polarisation on a cluster horizontal interface Q by $\|P\|=Q \cdot d$, which leads to: $\quad \Delta E=3 k_{B} T \cdot\left(\frac{e}{Q}\right)^{2}$

A value of $\|\mathrm{P}\|=5.10^{-29} \mathrm{C} . \mathrm{m}$ corresponds to $Q=0.16 e$ and (4) yields a $\Delta E$ value close to $60 \mathrm{meV}$ at $6 \mathrm{~K}$, in reasonable agreement with the experimental value of $90 \mathrm{meV}$. As can be inferred from (4), if $Q$ is independent of $\mathrm{t}_{\mathrm{Cr}}, \Delta E$ does not vary with clusters size. This model, which schematically reflects the main mechanism explaining the experimental low variation of $\Delta E$ with $\mathrm{t}_{\mathrm{Cr}}$ in Fig. 3, was checked by means of numerical simulations of $\Delta E$ using the same orthodox model as described above (Fig. 4). We supposed, due to polarisation, a constant surface electric charge $Q$ on the clusters. This simulation reproduces the trend experimentally observed as a function of $t_{C r}$ since it yields an almost constant $\Delta E$ value as well as $E_{1}$ and $E_{2}$ 
values close to the experimental ones -even though the linear variation with $t_{\mathrm{Cr}}$ is not fully reproduced-.

As a conclusion, we showed that the presence of a weak ferroelectric polarisation in strained $\mathrm{Cr}_{2} \mathrm{O}_{3}$ clusters has a dramatic influence on the Coulomb blockade energy of electrons tunnelling via those clusters: the increase of the capacitance of the clusters when increasing their size is compensated by a decrease of the dielectric constant $\varepsilon_{\mathrm{r}}$ due to the spontaneous electric polarisation of the clusters dielectric fraction. This leads to an almost constant energy difference between the first and second CB peaks as a function of the clusters area over a whole decade. This effect originates from the super-paraelectric property of the clusters assembly and the subsequent original dielectric constant behaviour that increases on decreasing the cluster size. This might also explain how measurements performed on average assemblies of clusters reveal well-defined Coulomb blockade: whatever the size involved in charge transfer, the $\mathrm{CB}$ energies involved vary little with size. We believe that this effect could be exploited to fabricate devices with a given $\mathrm{CB}$ energy, circumventing the unavoidable clusters size dispersion in large assemblies. Furthermore, a less naive model should take into account the electric - or magnetic- polarisation switch induced by a supplementary electron on a cluster. A model, similar to the ones performed by Udalov et al. ${ }^{[11,12,26]}$ would more accurately describe the involved physical mechanism and could perhaps explain the hysteretic conductance that we already reported at low temperature in those systems. ${ }^{[14]}$ For such purposes, the fabrication of 3-terminal devices will clearly provide better experimental insight, and possibly open the door to robust and multi-functional devices taking advantage of $\mathrm{CB}$ effects in clusters carrying a spontaneous electric polarisation. Obtaining such devices will require the increase of the intensity of $\mathrm{CB}$ peaks relative to the 
background conductance in $\mathrm{I}(\mathrm{V})$ curves: high quality and thicker $\mathrm{MgO}$ barriers could for instance favour the decrease of this background signal.

\section{Acknowledgements}

We thank the STnano clean room facility for technical support and partial support from the French National Research Agency (ANR) through the Programme d'Investissement d'Avenir under contract ANR-11-LABX-0058_NIE and ANR-17-EURE-0024 (B.K.) within the Investissement d'Avenir program ANR-10-IDEX-0002-02. We also are grateful to G. Weick for stimulating discussions.

\section{References:}

[1] Devoret, M. H. \& Grabert, H. in Single Charge Tunneling - Coulomb Blockade Phenomena in Nanostructures (eds Grabert, H. \& Devoret, M. H.) Ch. 1 (Plenum Press, New York, 1992).

[2] M. A. Kastner, Rev. Mod. Phys. 64, 849 (1992).

[3] Meirav, U. \& Foxman, E. B. Single-electron phenomena in semiconductors. Semiconductor Sci. Technol. 10, 255-284 (1995),

[4]A. Bernand-Mantel, P. Seneor, K. Bouzehouane, S. Fusil, C. Deranlot, F. Petroff and A. Fert, Nature Physics 5, 920 (2009)

[5] J.F. Scott, Science 315, 954 (2007)

[6] K. Ishikawa, K. Yoshikawa and N. Okada, Phys. Rev. B, 37, 5852 (1988)

[7] Räudiger, A., Schneller, T., Roelofs, A., Tiedke, S., Schmitz, T. and Waser, R. Appl. Phys. A 80, 1247 (2005).

[8] D. Pantel, S. Goetze, D. Hesse, M. Alexe, ACS Nano 5, 6032 (2011)

[9] V. Garcia, M. Bibes, L. Bocher, S. Valencia, F. Kronast, A. Crassous, X. Moya, S. EnouzVedrenne, A. Gloter, D. Imhoff et al. Science 327, 1106 (2010).

[10] S. Boyn, J. Grollier,, G. Lecerf,, B. Xu, N.Locatelli, S. Fusil, S. Girod, C. Carrétéro, K. Garcia, S. Xavier et al., Nature Comm. 8, 14736 (2017) 
[11] S. A. Fedorov, A. E. Korolkov, N. M. Chtchelkatchev, O. G. Udalov, and I. S. Beloborodov, Phys. Rev. B 89, 155410 (2014)

[12] O. G. Udalov, N. M. Chtchelkatchev,A. Glatz,and I. S. Beloborodov, Phys. Rev. B, 89 , 054203 (2014)

[13] L. Liu, V. Narayanan and S. Datta, Appl. Phys. Lett. 102, 053505 (2013)

[14] D. Halley, N. Najjari, H. Majjad, L. Joly, P. Ohresser, F. Scheurer, C. Ulhaq-Bouillet, S. Berciaud, B. Doudin, Y. Henry, Nat. Com. 5, 3167 (2014)

[15] M Hamieh, K D Dorkenoo, G Taupier, Y Henry and D Halley, J. Phys.: Condens. Matter 29, 205301 (2017)

[16] D. Halley, N. Najjari, F. Godel, M. Hamieh B. Doudin, and Yves Henry, Phys. Rev. B, 91214408 (2015)

[17] M. Mostovoy, A. Scaramucci, N. A. Spaldin, and K. T. Delaney, Phys. Rev. Lett, 105, $087202(2010)$.

[18] D.N. Astrov. Sov. Phys. JETP 11, 708 (1960)

[19] S. Sahoo, S. and C. Binek, Phil. Mag. Lett. 87, 259 (2007) and S. Mu and K. D. Belashchenko, Phys. Rev. Mat., 3, 034405 (2019)

[20] Koda, T., Mitani, S., Mizogushi, M. \& Takanashi, K., J. Phys. Conf. Ser. 266, 012093 (2011).

[21] Y. Nogi, H. Wang, F. Ernult, K. Yakushiji, S. Mitani and K. Takanashi, J. Phys. D : Appl. Phys. 40, 1242, (2007)

[22] L.E. Cross, Ferroelectrics 76, 241 (1987).

[23] D. Viehland, J. F. Li, S. J. Jang, L. E, Cross, and M. Wuttig, Phys. Rev. B 43, 8316 (1991)

[24] M. J. Polking, M.G Han, A. Yourdkhani, V. Petkov, C.F. Kisielowski, V.V. Volkov, Y. Zhu, G. Caruntu, A.P. Alivisatos and R. Ramesh, Nature Mater, 11, 700 (2012).

[25] P. H. Fang and W.S. Brower, Phys. Rev. 129, 1563 (1963) and M.A. Subramaniant, R.D. Shannon 1, B.H.T. Chaiz, M.M. Abraham and M.C. Wintersgill, Phys. Chem. Minerals 16, 741 (1989)

[26] O. G. Udalov, N. M. Chtchelkatchev, S. A. Fedorov, and I. S. Beloborodov, Phys. Rev. B 92, $205416(2015)$ 


\section{Figures:}

Figure 1. (a) Scheme of a tunnel junction with chromium oxide clusters embedded in a $\mathrm{MgO}$ tunnel barrier. The applied bias voltage induces an electric field $\mathbf{E}$. The electric spontaneous polarisation in each cluster (green arrows) fluctuates along E. (b) Scheme of a chromium wedge with a correlated variation of the clusters area $S$, keeping a constant height $d=2 \mathrm{~nm}$. (c) Tunnel junction conductance per junction surface unit $\mathrm{G} / \mathrm{A}$ as a function of $\mathrm{t}_{\mathrm{Cr}}$, measured under a $10 \mathrm{mV}$ bias voltage. A linear fit (red) is given showing the linear variation of the embedded $\mathrm{Cr}_{2} \mathrm{O}_{3}$ clusters area as a function of the deposited chromium thickness. Inset: Sketch of the vertical section of a chromium oxide cluster with a possible configuration of the metallic (blue)/ insulating(red) phases and of the electric charges and dipole.

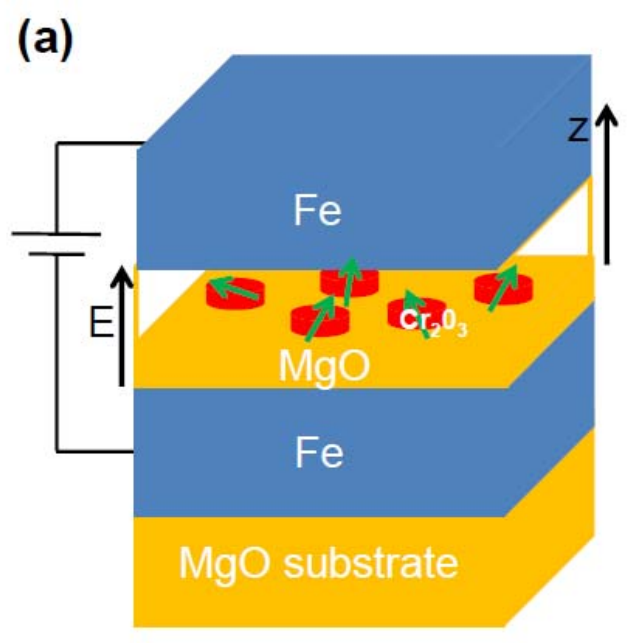

(b) Gradient of chromium nominal thickness $t_{c r}$

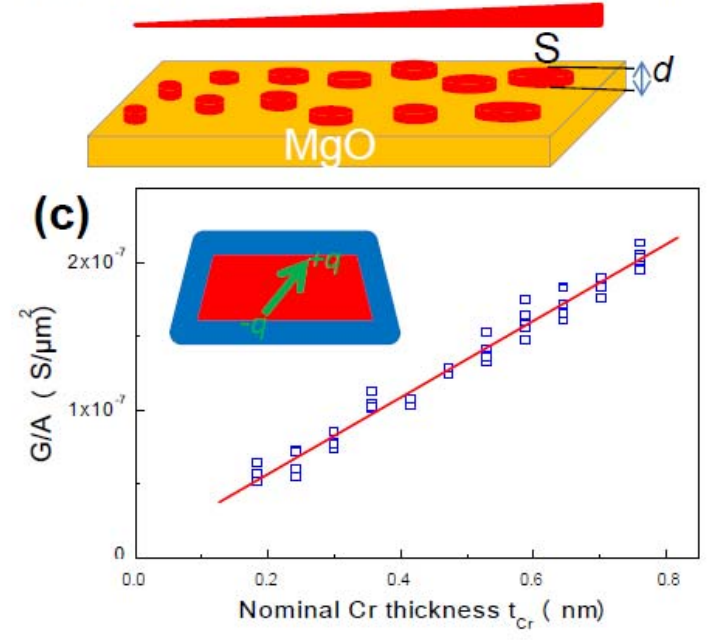


Figure 2. (a) $\mathrm{I}(\mathrm{V})$ curve and (b) $\frac{\partial^{2} I}{\partial V^{2}}$ measured at $6.2 \mathrm{~K}$ on a tunnel junction including $\mathrm{Cr}_{2} \mathrm{O}_{3}$ clusters with a nominal chromium thickness $\mathrm{t}_{\mathrm{Cr}}=0.71 \mathrm{~nm}$. The Coulomb blockade peak positions are marked by arrows. Inset: simulation of the current second derivative within the orthodox model described in the text, supposing an average permanent charge $\mathrm{Q}=0.28$ on each cluster.

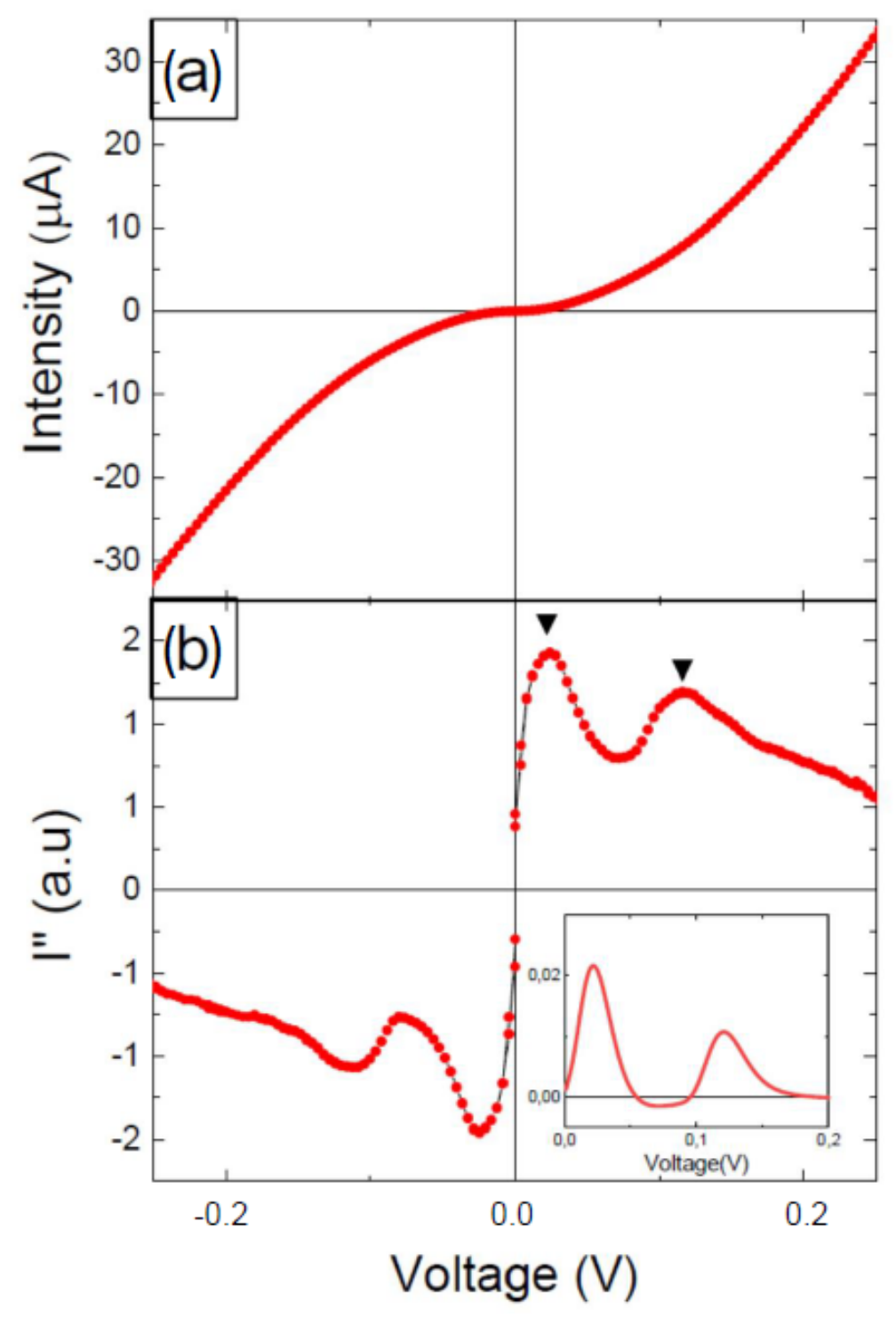


Figure 3. Energy of the measured first and second CB peaks in $\frac{\partial^{2} I}{\partial V^{2}}$ curves under a positive bias voltage, as a function of $\mathrm{t}_{\mathrm{Cr}}$. The difference $\Delta \mathrm{E}$ is given in blue. Linear fits are given for the three sets of data.

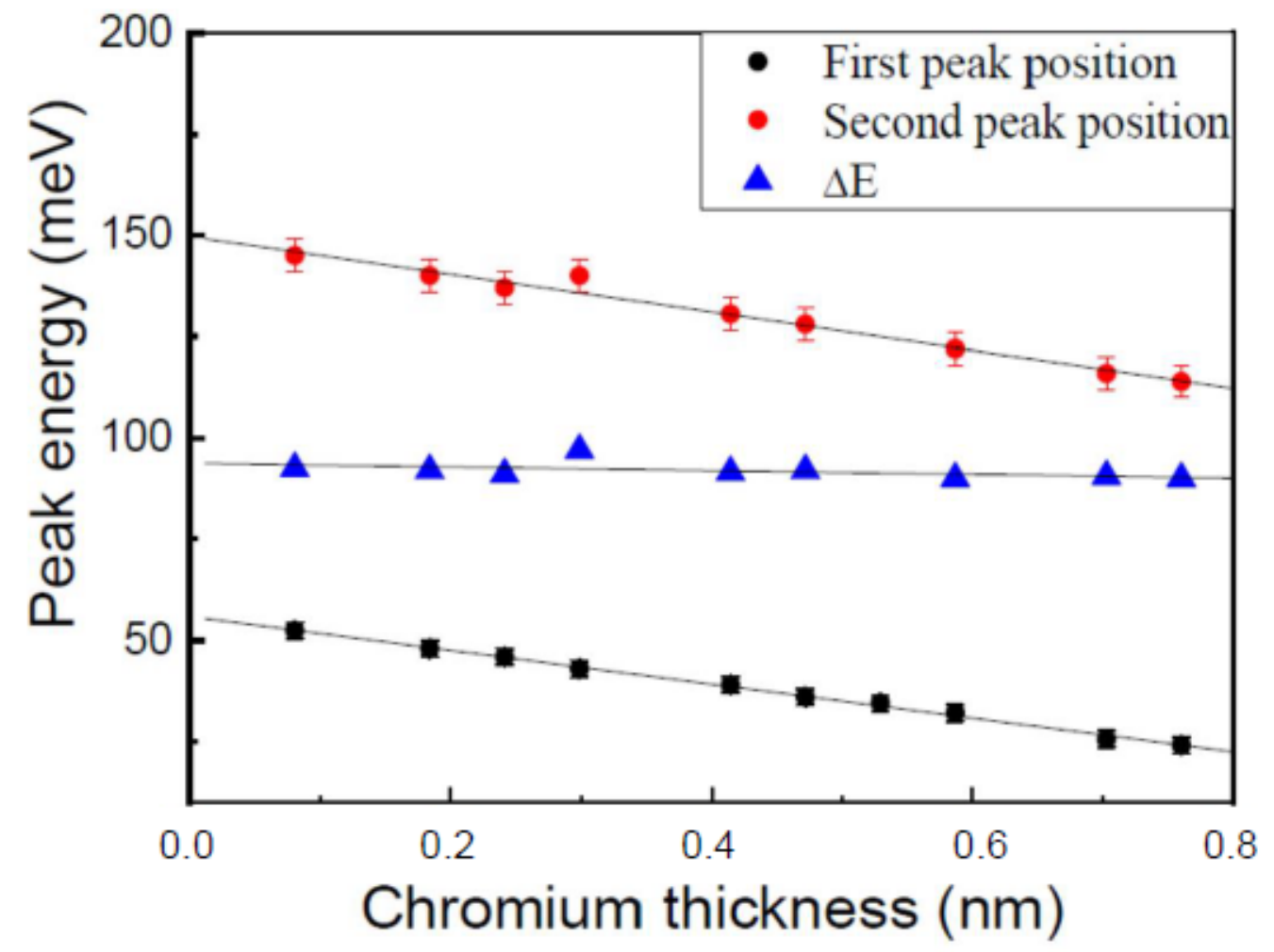

Figure 4: Calculation of the Coulomb blockade peak positions and of their difference $\Delta \mathrm{E}$, with a Gaussian distribution of the clusters diameter and a standard deviation of $1.2 \mathrm{~nm}$. The clusters density was taken equal to $1.310^{16} \mathrm{~m}^{-2}$, the $\mathrm{MgO}$ and $\mathrm{Cr}_{2} \mathrm{O}_{3}$ dielectric constants equal to respectively 9.8 and 5.5. (a) Supposing a $\mathrm{Q}_{0}$ environment charge on the clusters following a Gaussian distribution centred on zero with $\Delta \mathrm{Q}_{0}=0.2 \mathrm{e}, \Delta \mathrm{E}$ noticeably decreases with $\mathrm{t}_{\mathrm{Cr}}$.(b) Supposing a constant electric polarisation in the clusters, i.e. that the surface charge $Q$ is centred on a constant value equal to $0.28 \mathrm{e}, \Delta \mathrm{E}$ is nearly constant. 


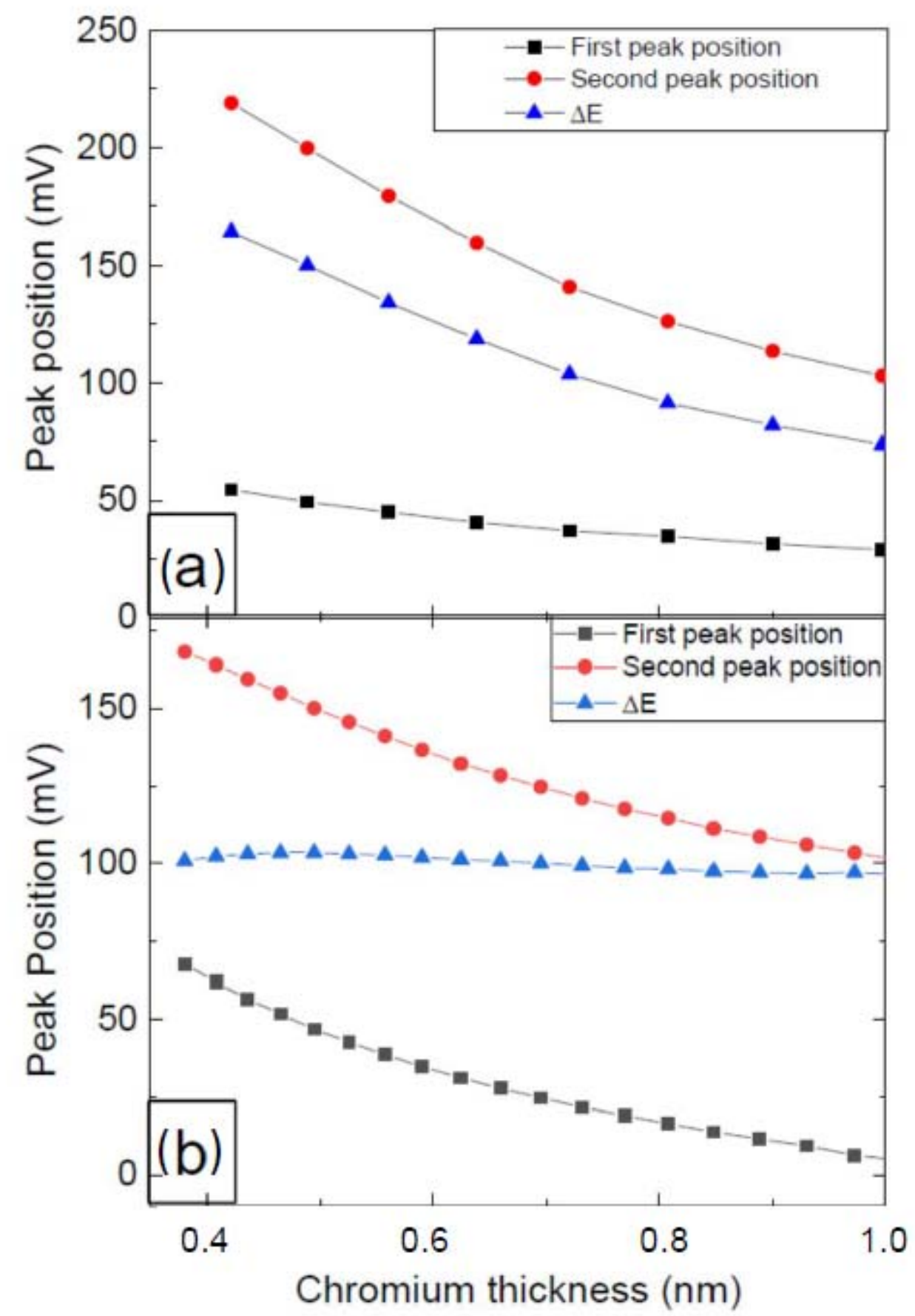

